\title{
Formulation of Nano and Micro PLGA Particles of the Model Peptide Insulin: Preparation, Characterization, Stability and Deposition in Human Skin
}

\author{
F. Wang, Y. Chen ${ }^{*}$ and H.A.E. Benson
}

School of Pharmacy, Curtin University of Technology, Perth, Western Australia

\begin{abstract}
The primary objective of this study was to develop a particulate formulation for peptide delivery that would provide enhanced peptide stability, controlled release and the potential for targeting to specific tissues. Biodegradable hydrophobic particles were prepared from poly (D,L-lactide-co-glycolide) (PLGA) by both solvent evaporation and solvent diffusion methods. Bovine insulin was chosen as a model peptide for formulation development and evaluation. By forming a complex between insulin and protamine, $50 \%$ incorporation of the model peptide in PLGA particles was achieved and a sustained release of insulin was observed over one week with improved stability of insulin in the PLGA matrix. The formation of an insulin-protamine complex and the method of manufacture are important determinants of the physicochemical characteristics of the particles formed. Preliminary evaluation of the deposition of the particles within skin was determined by fluorescent images following topical application to excised human skin. Microparticles with size above 7 $\mu \mathrm{m}$ remained on the surface of the skin. Nanoparticles $(<1 \mu \mathrm{m})$ showed permeation into the viable epidermis and dermis with deposition concentrated around the hair follicles and sebaceous glands.
\end{abstract}

Keywords: Microparticles, nanoparticles, skin deposition, insulin, PLGA, FESEM.

\section{INTRODUCTION}

Particulate carrier systems have been investigated for localized skin therapy to minimize systemic exposure and maximize local drug concentrations. They have been shown to improve the permeation of some drugs by targeting to specific sites in the skin (primarily the hair follicles) and prolonging local drug concentration [1]. Particulate carriers can also offer potential advantages by enhancing drug stability and reducing the skin irritation associated with some drugs. This could offer significant advantages for many cosmetic/anti-ageing applications of antioxidants which tend to be difficult to formulate as stable topical products. In addition, a number of peptides with potential therapeutic use in the skin have been identified [2]. Nanoparticles could provide a means of protecting these peptides from skin peptidases and targeting their delivery to or within the skin.

Deposition of nanoparticles within the skin appears to be dependant on the particle size and possibly also the nature of the particle formulation [3]. Alvarez-Roman et al. examined cutaneous localization of FITC-labeled non-biodegradable polystyrene nanoparticles using confocal laser scanning microscopy (CLSM) [4]. The CLSM images showed preferential accumulation of 20 and $200 \mathrm{~nm}$ polystyrene nanoparticles in follicular openings in a time and size-dependent fashion with no evidence of permeation of nanoparticles in the non-follicular region. Recently, Gamer et al. (2006) reported accumulation of zinc oxide and titanium dioxide nanoparticles in hair follicles [5]. Another group, Cross et al. reported less than $0.03 \%$ of the applied $\mathrm{Zn}$ content penetrated the epidermis and minimal nanoparticle penetration through human

*Address correspondence to this author at the School of Pharmacy, Curtin University of Technology, GPO BOX U1987, Perth, WA 6845, Australia. Tel: +61 89266 2738; Fax: +61 89266 2769; E-mail: y.chen@curtin.edu.au epidermis occurred when a transparent, nanoparticulate $\mathrm{ZnO}$ sunscreen formulation was tested using Franz-type diffusion cells [6]. Lademann and co-workers (2007) also demonstrated that dye loaded poly DL, lactide-co-glycolide (PLGA) nanoparticles $(320 \mathrm{~nm})$ deposited and remained in the hair follicles for up to 10 days whilst a non-particulate form remained only up to 4 days [1].

Encapsulation of therapeutic peptides in biocompatible and biodegradable polymeric nanoparticles is a commonly used strategy to bypass the early degradation of peptides in vivo [7]. Polymeric nanoparticles are solid colloidal carriers ranging in size from 10 to $1000 \mathrm{~nm}$, prepared from various materials including lipids, synthetic and natural polymers. Among those, poly (D,L-lactide-co-glycolide) (PLGA) has been extensively used as it is a FDA approved biodegradable and biocompatible material [8]. Although PLGA nanoparticles have been widely considered as the preferred choice for the delivery of peptides because of biocompatibility and biodegradability, formulation of water-soluble peptides/proteins into PLGA nanoparticles still remains a challenge. This is largely because PLGA is not water soluble and encapsulation of water soluble peptides into PLGA particles requires either the double emulsion $(\mathrm{w} / \mathrm{o} / \mathrm{w})$ solvent evaporation method or a solvent diffusion/nanoprecipitation method. The former involves w/o interfaces which are a potential cause of protein instability and aggregation [9]. Moreover, both methods also have the shortcoming of marked reduction of peptide loading capacity. This occurs due to the large surface area to volume ratio and consequent rapid migration which results in rapid loss of drug into the external phase, during formation of the nanoparticles $[10,11]$. In recent years, approaches such as using protein-fatty acid complexes via hydrophobic ion pairing have been used to enhance encapsulation as has been reported for salmon calcitonin [12]. Cui and colleagues described the use of insulin-phospholipid (soybean phosphatidylcholine, SPC) complex to improve the liposolubility of 
insulin and its incorporation in PLGA nanoparticles for oral delivery of insulin [13]. However, the use of water soluble polyanion and polycation to form complexes that increase the water soluble peptide incorporation into PLGA nanoparticle has not been investigated.

The aim of this study was to develop a particulate peptide delivery system capable of high peptide loading, controlled release and improved stability. In particular, the optimization of formulation and method of manufacture to achieve these goals was determined. Preliminary investigation of the deposition within the skin was also investigated. Insulin was chosen as a model polypeptide since its structure, stability and physicochemical characteristics have been extensively studied and it is therefore a good model of a chemically and physically unstable peptide.

\section{MATERIALS AND METHODS}

\section{Materials}

PLGA (Purasorb ${ }^{\circledR}$ PDLG 85/15, Mw: 15,600 Da) was purchased from Purac Biochem, Gorinchem, Netherlands. Insulin from bovine pancreas ( $\geq 27$ UPS units/mg (HPLC) powder), protamine from salmon and phosphate buffered saline (PBS, $10 \mathrm{mM}, \mathrm{pH}$ 7.4) tablets were obtained from Sigma-Aldrich Co. (St. Louis, MO, USA). Poly (vinyl alcohol) (PVA, 80\% hydrolyzed, Mw: 9,000-10,000 Da) was purchased from Aldrich Chemical Co. (Milwaukee, USA). Rhodamine 6G (R6G, Mw: 479) was supplied by SigmaAldrich, UK.

\section{Methods}

\section{Preparation of Nanoparticles by Insulin-Protamine Com- plexation with a Solvent Evaporation Method}

Insulin was incorporated into PLGA nanoparticles at a theoretical loading of $1.64 \% \mathrm{w} / \mathrm{w}$. Briefly, $5 \mathrm{mg}$ insulin, 100 $\mu \mathrm{g}$ R6G and $0.5 \mathrm{mg}$ protamine were dissolved in $0.75 \mathrm{~mL}$ $0.01 \mathrm{M}$ hydrochloric acid $(\mathrm{HCl})$ solution. $0.75 \mathrm{~mL}$ of this solution was mixed with $0.75 \mathrm{~mL} 10 \mathrm{mM}$ phosphate buffer ( $\mathrm{pH}$ 7.8) to form a nano-suspension. This suspension was poured into $10 \mathrm{~mL}$ of dichloromethane (DCM) containing $300 \mathrm{mg}$ PLGA and sonicated in an ice bath for 2 min at output level $10(50 \mathrm{~W})$ for $2 \mathrm{~min}$ (Microson $^{\mathrm{TM}}$, ultrasonic cell disruptor, equipped with P-3 microprobe, Heat Systems Inc. USA) to form a W/O emulsion. This primary emulsion was added to $100 \mathrm{~mL}$ of $1 \%$ PVA solution and sonicated in an ice bath at output level 10 for 2 min to form a W/O/W multiple emulsion in a $250 \mathrm{~mL}$ beaker. This multiple emulsion was stirred at room temperature by a magnetic stirrer for $3 \mathrm{~h}$ to evaporate the organic solvent. Empty particles were prepared using the same procedure without insulin.

After the formation of PLGA particles, $40 \mathrm{~mL}$ of the particle suspension was centrifuged at $15,000 \mathrm{rpm}$ (Allegra ${ }^{\mathrm{TM}}$ 64R centrifuge, Beckman Coulter, Inc., CA, USA) for 15 min, the particles were washed with $10 \mathrm{~mL}$ water and resuspended in 2-3 mL water after removal of supernatant by centrifugation at $15,000 \mathrm{rpm}$ for $15 \mathrm{~min}$. The final particle suspension was frozen at $-40^{\circ} \mathrm{C}$ overnight and then lyophilized (Dynamic FD3, Dynamic Engineering Pty. Ltd., Australia) for $24 \mathrm{~h}$ before being collected and stored for further use.
Microparticle Preparation by Insulin-Protamine Complexation with a Solvent Diffusion Method

Insulin $5 \mathrm{mg}$, R6G $100 \mu \mathrm{g}$ and protamine $0.5 \mathrm{mg}$ were dissolved in $0.75 \mathrm{~mL} 0.01 \mathrm{M} \mathrm{HCl}$ solution. $0.75 \mathrm{~mL}$ of this solution was mixed with $0.75 \mathrm{~mL}$ phosphate buffer $(\mathrm{pH} 7.8)$ to form a suspension. The suspension was poured into $10 \mathrm{~mL}$ of ethanol/acetone $(4 / 6, \mathrm{v} / \mathrm{v})$ containing $300 \mathrm{mg}$ PLGA. This was added to $100 \mathrm{~mL}$ of $1 \%$ PVA solution and stirred at room temperature by a propeller stirrer (RZR 2102 control with three blades, Heidolph Instruments, Germany) at 405 $\mathrm{rpm}$ for $1 \mathrm{~h}$ to evaporate the organic solvent. The suspension was filtered through a $53 \mu \mathrm{m}$ mesh to remove aggregates. Empty particles were prepared in the same fashion without insulin.

\section{Particle Morphology}

Morphological evaluation of the particles was performed using a field emission scanning electron microscope (FESEM: Leo Supra 55 Variable Pressure FESEM, Germany). The samples were gold coated before examination by the FESEM.

\section{Particle Size and Zeta-Potential}

Nanoparticle size was determined by photon correlation spectroscopy (PCS) using a Zetasizer 3000HS (Malvern Instrument, UK). The measurement was performed at $25^{\circ} \mathrm{C}$ with a detection angle of $90^{\circ}$ and the raw data was subsequently correlated to $\mathrm{Z}$ average mean size using a cumulative analysis by the Zetasizer 3000 HS software package. Each sample was measured ten times. Microparticle size was measured using a HIAC particle counter (Hiac Royco 8000, Pacific Dynamics Pty. Ltd., USA). Two to three drops of the particle suspension were added into $100 \mathrm{~mL}$ water and stirred at about $50 \mathrm{rpm}$ for 30 seconds. Triplicate measurements were performed for each sample.

The zeta potential of particles was determined by Laser Doppler Anemometry using a Zetasizer 3000HS. All analyses were performed on samples appropriately diluted with filtered de-ionised water. For each sample, the mean \pm standard deviation of three determinations was established.

\section{Determination of Particles Yield}

Upon the formation of particles, a volume of nano- or microparticle suspension was centrifuged at 15,000 rpm for 15 min to remove the supernatant. The particles were washed once with water, separated from the supernatant and dried at $80^{\circ} \mathrm{C}$ for $24 \mathrm{~h}$ to a constant weight. The original weight of centrifuge tube and the weight of the centrifuge tube containing dry particles were recorded. A tube with the same volume of water was used as a control. The yield was calculated by the following equation.

$$
\text { yield }(\%)=\left(\frac{\text { weight of particles }}{\text { total amount of polymers and drug used }}\right) \times 100
$$

Determination of Insulin Loading and Entrapment Efficiency of PLGA Particles

15 to $30 \mathrm{mg}$ of PLGA micro- or nanoparticles loaded with insulin was accurately weighed, dissolved in $1 \mathrm{~mL}$ of acetonitrile $(\mathrm{ACN})$ and the insulin extracted into $2 \mathrm{~mL}$ of $\mathrm{HCl} 0.01 \mathrm{M}$. The suspension was centrifuged at 15,000 rpm for $15 \mathrm{~min}$ and the supernatant analyzed for insulin content 
by the HPLC method described below. The drug loading and entrapment efficiency were expressed as follows; the entrapment efficiency was calculated as the percentage of insulin loading over theoretical loading.

Loading $(\%)=\left(\frac{\text { Total amount of insulin loaded }}{\text { Weight of particles } \times(1-P V A \text { content })}\right) \times 100$

Entrapment efficiency $\quad(\%)=\left(\begin{array}{lll}\text { Formulated insulin loading } \\ \hline \text { Theoretical insulin loading }\end{array}\right) \times 100$

\section{Determination of R6G Loading and Entrapment Efficiency}

A volume of nanoparticle suspension (one fifth of the total suspension) was centrifuged at $15,000 \mathrm{rpm}$ for $15 \mathrm{~min}$, the first supernatant collected and the particles washed with water. After a second centrifugation the second supernatant was collected. The unloaded R6G in both supernatants (named as Free R6G) was measured by UV-VIS spectrophotometry (UV-1201, Shimadzu Corporation) at $524 \mathrm{~nm}$. The samples were the diluted appropriately and analyzed by a fluorometer (Cary Eclipse, Varian Instruments, USA) with a slit width of $5 \mathrm{~nm}$ and excitation and emission wavelengths at $527 \mathrm{~nm}$ and $550 \mathrm{~nm}$, respectively. The loading and entrapment efficiencies were calculated as follows:

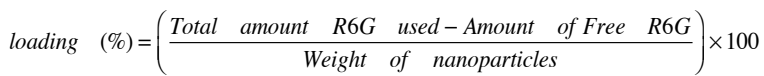

Entrapment Efficiency $(\%)=\left(\begin{array}{ccccc}\text { Total amount } & R 6 G-\text { Amount } & \text { of Free } & R 6 G \\ \text { Total } & \text { amount } & \text { R6G } & \text { used }\end{array}\right) \times 100$

\section{Determination of PVA Content}

The content of PVA in insulin-loaded PLGA particles was determined by a validated colorimetric method based on the formation of a coloured complex between two adjacent hydroxyl groups of PVA and an iodine molecule [14]. Briefly, $1 \mathrm{~mL}$ of $5 \mathrm{mg} / \mathrm{mL}$ of insulin loaded PLGA particles, prepared from freeze dried particles, was centrifuged at $10,000 \mathrm{rpm}$ for $10 \mathrm{~min} .0 .5 \mathrm{~mL}$ of the supernatant was diluted with $5 \mathrm{~mL}$ distilled water, followed by addition of 3 $\mathrm{mL}$ boric acid $(3.8 \% \mathrm{w} / \mathrm{v})$ and $0.6 \mathrm{~mL} 0.1 \mathrm{M}$ iodine solution and then made up to $10 \mathrm{~mL}$ with distilled water. The absorbance of the samples was measured by UV-VIS spectrophotometry at $690 \mathrm{~nm}$. A standard plot of PVA was prepared under identical conditions with a concentration range from 0 to $35 \mu \mathrm{g} / \mathrm{mL}$. As a control, 4.5 and $80 \mu \mathrm{g} / \mathrm{mL}$ insulin solutions were prepared under the same conditions and analysed.

In Vitro Release Study of PLGA Particles Loaded with Insu$\underline{\text { lin }}$

$15 \mathrm{mg}$ of freeze dried PLGA micro- or nanoparticles were weighed, resuspended in $1.5 \mathrm{~mL}$ of PBS at $\mathrm{pH} 7.4$ and incubated at $37^{\circ} \mathrm{C}$. Due to the instability of insulin in the stirring medium [15], the release study was carried out under gentle agitation. $0.05 \%$ sodium azide was added into the buffer as a preservative against the growth of bacteria, and in order to counteract the adsorption of insulin to glass $0.2 \%$ BSA was included in PBS.

The supernatants were isolated by centrifugation at $15,000 \mathrm{rpm}$ for $7 \mathrm{~min}$ and replaced by an equal volume of fresh medium in time intervals of first hour and then every $24 \mathrm{~h}$ up to 7 days. The concentration of insulin in the super- natants was analysed by the validated HPLC method described below. Triplicate samples were performed for each batch. The empty PLGA particles and insulin standard in PBS were used as controls. They were incubated at $37^{\circ} \mathrm{C}$ and analysed by the same method.

\section{$\underline{\text { Insulin Stability }}$}

Insulin stability was determined in the extraction medium of ACN: $0.01 \mathrm{M} \mathrm{HCl}(1: 2 \mathrm{v} / \mathrm{v})$. Insulin of $150 \mu \mathrm{g} / \mathrm{mL}$ in the solvent was analysed immediately after preparation as well as after storage at room temperature for 1, 3 and 7 days and after storage at $37^{\circ} \mathrm{C}$ for 3 and 7 days. Insulin potency was calculated as a percentage of insulin peak area over the total area of peaks including insulin and degradation products. Results are reported as mean \pm standard deviation (SD).

Insulin Stability in Release Medium

Triplicate samples with concentrations of $120 \mu \mathrm{g} / \mathrm{mL}$ of insulin in PBS (pH 7.4, containing $0.05 \%$ sodium azide and $0.2 \%$ BSA) were analyzed immediately after preparation as well as after 3 and 7 days at $37^{\circ} \mathrm{C}$. HPLC analysis was performed after suitable sample dilution. Insulin potency was calculated as a percentage of insulin peak area over the total area of peaks including insulin and degradation products.

\section{Insulin Stability Inside PLGA Particles}

Insulin stability within particles during in vitro release was assessed by HPLC. After in vitro release, the suspension was centrifuged at $15,000 \mathrm{rpm}$ for $15 \mathrm{~min}$ to remove the supernatant. The encapsulated non-released insulin was extracted by the method described for the measurement of insulin loading of particles.

Insulin potency was calculated as a percentage of insulin peak area over the total area of peaks including insulin and degradation products. Triplicate samples were carried out for each batch. Duplicate batches were assessed. Insulin solution $(120 \mu \mathrm{g} / \mathrm{mL}$ in PBS) was used as a control.

\section{HPLC Analysis of Insulin}

The insulin HPLC analysis method was developed from a reported method [16]. Insulin was analysed by reversed phase-HPLC (Agilent LC1100 Series, Agilent Technologies; Germany) using a Jupiter $5 \mu \mathrm{m}$ C18 $300 \AA$ column $(150 \times 4.60$ $\mathrm{mm}$; Phenomenex, Torrance, CA) maintained at $25^{\circ} \mathrm{C}$ and a mobile phase consisting of $0.1 \%$ trifluoroacetic acid (TFA) in water and 0.1\% TFA in ACN in a ratio of 70.5:29.5 (v/v) in the isocratic mode. The solvent flow rate was $1 \mathrm{~mL} / \mathrm{min}$ and peaks were detected at $205 \mathrm{~nm} .10 \mu \mathrm{L}$ and $50 \mu \mathrm{L}$ injection volumes were used for insulin in mediums of ACN: $0.01 \mathrm{M} \mathrm{HCl}(1: 2, \mathrm{v} / \mathrm{v})$ and PBS $(\mathrm{pH} 7.4,10 \mathrm{mM})$ respectively. This insulin assay method was fully validated.

\section{Skin Permeation Study}

Human skin obtained from abdominoplasty surgery at Perth hospitals was used (under current approval by the Curtin University Human Research Ethics Committee). The freshly obtained skin was frozen at $-20^{\circ} \mathrm{C}$ immediately upon reception. Prior to use, the whole skin was thawed, the fat carefully removed and the skin rinsed with water. Franz-type diffusion cells with diffusion area of $1.23 \mathrm{~cm}^{2}$ were used to investigate the permeation of micro- and nanoparticles through the skin. The whole skin was placed between the 
receptor and donor compartments of the cell with the stratum corneum side facing the donor compartment. The receptor compartment was filled with $3 \mathrm{~mL}$ PBS (10mM, pH 7.4, containing $0.05 \%$ sodium azide, $0.2 \%$ BSA), maintained at $37^{\circ} \mathrm{C}$ and stirred continuously. After $30 \mathrm{~min}$ the integrity of the skin was tested by transepidermal water loss (TEWL) before application of the particle suspension on the skin.

PLGA particles $(5 \mathrm{mg}$ ) were weighed and re-suspended in $1 \mathrm{~mL}$ water. The suspension was placed in the donor compartment which was covered by a glass cover-slip. The receptor phase (PBS 10mM, pH 7.4, containing $0.05 \%$ sodium azide, $0.2 \% \mathrm{BSA}$ ) was maintained at $37^{\circ} \mathrm{C}$ in order to ensure a surface skin temperature of $32^{\circ} \mathrm{C}$. After the solution in the receptor chamber was gently mixed, $300 \mu \mathrm{L}$ samples from the receptor chamber were removed at intervals over an $8 \mathrm{~h}$ period for a total of $24 \mathrm{~h}$ and analysed by HPLC for insulin content and by fluorimetry for R6G content. The removed samples were replaced by an equal volume of fresh buffer at $37^{\circ} \mathrm{C}$.

PLGA particles loaded with insulin or R6G with different size and charge, prepared by the methods described above, were used in the skin permeation study to investigate the effect of physicochemical properties of particulate carriers. As a control, R6G solution with a concentration of $1 \mu \mathrm{g} / \mathrm{mL}$ was also applied to the skin. Skin with water on the surface was used to evaluate the intrinsic fluorescence of the skin.

At the end of the permeation experiment the skin was removed, rinsed twice with water and the middle was cut into square shaped pieces. The cut direction was from the dermis to the stratum corneum and the knife was thoroughly cleaned after each cut to prevent contamination of the particles from the skin surface. Each piece of the skin was embedded in a small tissue embedding mold with Tissue-Tek O.C.T. compound embedding medium (ProSciTech, Australia) and then rapidly frozen at $-80^{\circ} \mathrm{C}$. After fixation, the frozen skin block was cut vertically from the dermis to the stratum corneum in $10 \mu \mathrm{m}$ sections by a Cryomicrotome (Leica CM 3050 S, Germany). The sections were collected with microscopy slides and examined under a Zeiss Axioscope II fluorescence microscope with excitation and emission wavelengths at $546 \mathrm{~nm}$ and $590 \mathrm{~nm}$ respectively. An AxioCam digital camera with Axiovision software was used to record pictures of various skin samples.

\section{RESULTS AND DISCUSSION}

\section{Stability of Insulin}

Like many peptides, insulin is unstable in an acid environment and transforms into side-products such as the hy- drolysis product desamido-insulin [16]. In order to determine if the intrinsic instability of insulin could distort the results of the drug loading and in vitro release studies, insulin stability in the drug extraction and release mediums was investigated. The stability of insulin within PLGA particles was also evaluated.

The stability of insulin in the acidic extraction medium of ACN:0.01M HCl $(1: 2, v / v)$ is presented in Tables 1 and 2. As expected, insulin degraded faster at $37^{\circ} \mathrm{C}$ than at room temperature, with almost $40 \%$ of insulin transformed into degradation products after 7 days storage at $37^{\circ} \mathrm{C}$. Insulin was also unstable at room temperature with an increase of $4.24 \%$ in degradation products observed after 3 days, but only a $1.67 \%$ increase in degradation products after $24 \mathrm{~h}$. Consequently analysis of freshly prepared samples was conducted within $24 \mathrm{~h}$ and samples were stored at low temperature prior to analysis.

Insulin stability in the release medium (PBS containing $0.2 \%(\mathrm{w} / \mathrm{v}) \mathrm{BSA}$ and $0.05 \%$ sodium azide) was evaluated at $37^{\circ} \mathrm{C}$. Less than $1 \%$ increase of insulin degradation was detected after 3 days (Table 1). After 7 days storage at $37^{\circ} \mathrm{C}$, the percentage of insulin degradation was increased from initial level of $3.60 \%$ to $5.63 \%$, with about $2 \%$ increase in degradation products. These results are in agreement with that previously reported for insulin instability under similar conditions [15]. Hence the stability of insulin in the release medium has little effect on the in vitro release study data. Comparing the stability of insulin in the extraction medium and release medium, it can be concluded that insulin is unstable in acid conditions and relatively more stable in neutral conditions

It has been reported that PLGA microparticles can form acidic conditions inside the particles due to the acidic characteristics of the PLGA degradation product and this may accelerate the degradation of bovine insulin $[17,18]$. In order to determine if the PLGA matrix affects the stability of insulin incorporated into the PLGA particles after complexation with protamine, a study of the stability of insulin in PLGA particles was performed.

Stability of insulin in PLGA particles was assessed by extracting nanoparticles prepared by complexation of insulin with protamine (batch C \& D) which had completed 7 days of the release study (Table 2 ). The extracts were then analysed by HPLC. As a control, insulin standard in the release medium was collected after an equal length of time at $37^{\circ} \mathrm{C}$ and analysed by the same assay method. After 7 days storage at $37^{\circ} \mathrm{C}$, the amount of insulin degradation in particles increased from the initial level of $3.08 \pm 1.14 \%$ to $5.33 \pm 0.76 \%$, not significantly different from that of the control $(5.63 \%)$.

Table 1 Insulin Stability in the Solution of ACN:0.01M HCI (1:2, v/v) and Release Medium

\begin{tabular}{|c|c|c|c|c|c|}
\hline \multirow{2}{*}{ Medium } & \multirow{2}{*}{ Temperature } & \multicolumn{4}{|c|}{ Insulin Potency $(\%)^{*}$} \\
\hline & & Day 0 & Day 1 & Day 3 & Day 7 \\
\hline \multirow{2}{*}{$\mathrm{ACN}: 0.01 \mathrm{M} \mathrm{HCl}(1: 2 \mathrm{v} / \mathrm{v})$} & Room Temperature & 96.38 & 94.71 & 92.14 & 87.40 \\
\hline & $37^{\circ} \mathrm{C}$ & 97.00 & -- & 86.40 & 57.59 \\
\hline Release Medium $^{* *}$ & $37^{\circ} \mathrm{C}$ & $96.34 \pm 0.25$ & -- & $95.41 \pm 0.38$ & $93.97 \pm 0.76$ \\
\hline
\end{tabular}

${ }_{* *}^{*}$ Insulin potency was calculated as a percentage of insulin peak area over the total area of peaks including insulin and degradation products 
Therefore, it can be concluded that the current formulation containing insulin-protamine complex has no effect on insulin stability over the period of 7 days, which is an improvement in insulin stability compared to direct incorporation of insulin in PLGA matrix as reported previously by Uchida et al. [18]. They reported that over $10 \%$ of bovine insulin in PLGA matrix was converted to a degradation product after 7 days release test in $\mathrm{pH} 7.4$ Tris buffer. The difference between the data obtained in our study and that in the literature can be attributed to the different formulation techniques employed. In Uchida's study, PLGA microparticles were prepared by an oil-in-oil emulsion solvent evaporation method whilst the current formulation comprised insulin complexation with protamine before incorporation into the PLGA matrix. This suggests that the formation of the insulin/protamine complex can effectively stabilize insulin leading to higher stability of insulin in the PLGA matrix.

\section{Formulation of Insulin Loaded PLGA Micro- and Nanoparticles}

The formation of insulin-protamine complex has been used in the formulation of commercial insulin suspensions for injection and has been demonstrated to prevent degradation in aqueous solution and provide a sustained pharmacological effect after subcutaneous administration [19]. Insulin is negatively charged while protamine is positively charged at neutral $\mathrm{pH}$. Fine crystals formed immediately after these two oppositely charged compounds were mixed at $\mathrm{pH}$ 5.75.8. Despite the benefits of protamine inclusion, the complex is not expected to deliver insulin transepidermally due to its hydrophilic characteristics and large size, both of which are negative for skin permeation. The encapsulation of peptide complexes in particulate systems such as hydrophobic polymeric micro- and nanoparticles, could be an alternative to improve the permeation of peptides into skin as micro/nanoparticles can be used to mask the intrinsic properties of therapeutic agents and deliver them into the skin via appendages $[1,4]$.

\section{Physicochemical Characteristics of PLGA particles}

The morphology of the micro- and nanoparticles was examined by FESEM as shown in (Fig. 1). As indicated in the image, the surface of the nanoparticles prepared by the solvent evaporation method is less spherical with evidence of some nano-structures adsorbed to the particle surface. Particles prepared by the solvent diffusion method were larger in size with a spherical and solid structure, also with some nano-structures adsorbing to the particle surface. It is likely that such structures are insulin-protamine complex which absorbed on the particle surface. (a)

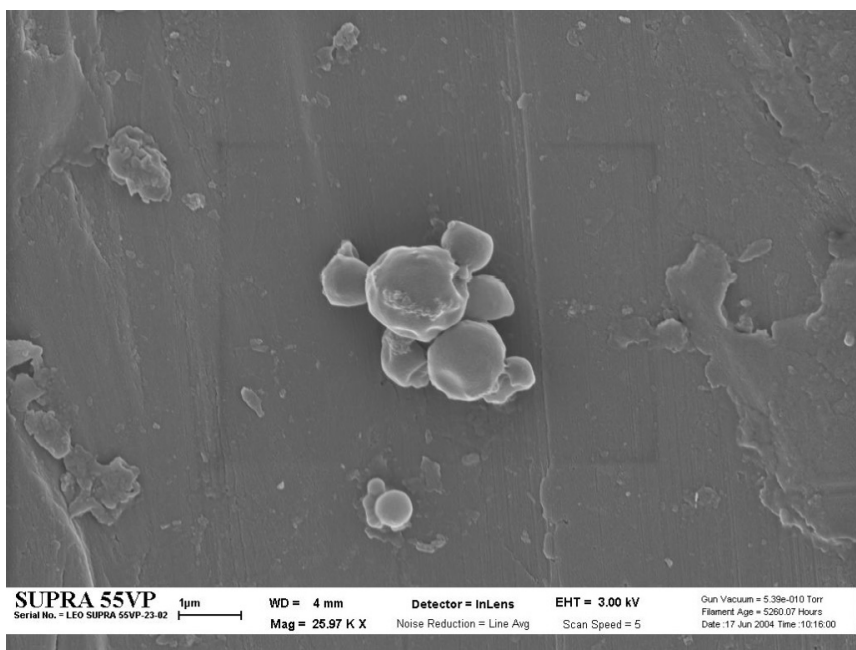

(b)

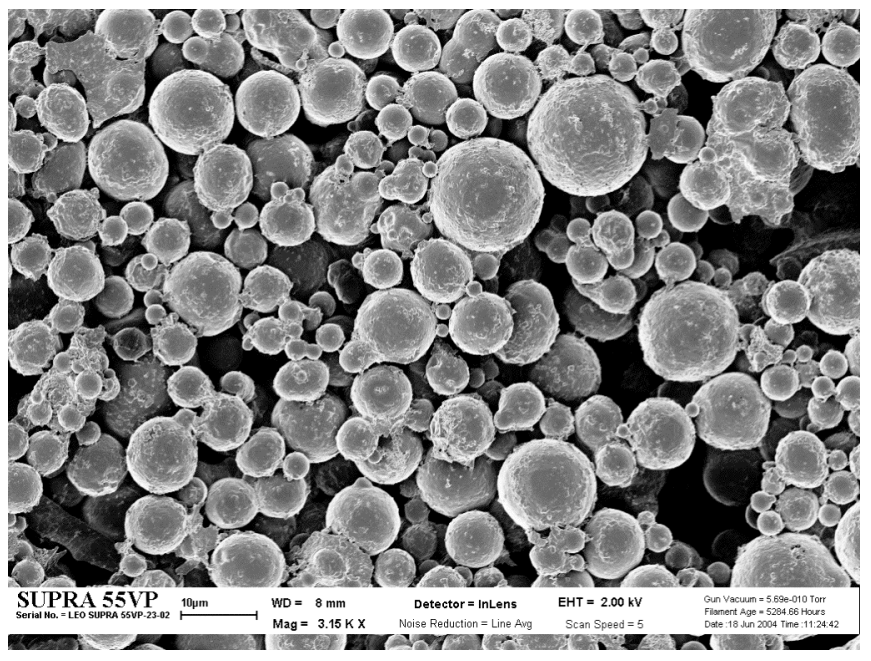

Fig. (1). (a) FESEM image of insulin-loaded PLGA nanoparticles prepared by protamine-insulin complex and solvent evaporation method. (b) FESEM image of insulin loaded PLGA nanoparticles prepared by protamine-insulin complex and solvent diffusion method.

The physical characteristics of insulin loaded PLGA micro- and nanoparticles are shown in Table 3. Duplicate samples were prepared under the same conditions (Batch A\&B, Batch C\&D, Batch E\&F, Batch G\&H). Insulin-loaded particles prepared by the solvent diffusion method were larger $(7.27-8.47 \mu \mathrm{m})$ than those prepared by the solvent evaporation method (274-472.5 nm, Table 3). The incorporation of R6G had minimal impact on particle size. The large particles (micron range) produced by the solvent diffusion method

Table 2. Insulin Stability Inside PLGA Particles Over 7 Days at $37^{\circ} \mathrm{C}$

\begin{tabular}{|c|c|c|c|}
\hline \multirow{2}{*}{ Sample } & Time (Day) & Insulin Potency (\%) & Total Degradation Products (\%) \\
\hline \hline \multirow{2}{*}{ Insulin loaded PLGA particles } & 0 & $96.92 \pm 1.14$ & $3.08 \pm 1.14$ \\
\cline { 2 - 4 } & 7 & $94.67 \pm 0.76$ & $5.33 \pm 0.76$ \\
\hline \multirow{2}{*}{ Control } & 0 & 96.38 & 3.62 \\
\cline { 2 - 4 } & 7 & 94.37 & 5.63 \\
\hline
\end{tabular}

Insulin potency was calculated as a percentage of insulin peak area over the total area of peaks including insulin and degradation products, $\mathrm{n}=6$. 
Table 3. Characteristics of Insulin Loaded PLGA Particles Made by Insulin-Protamine Complex and Solvent Evaporation and Solvent Diffusion Method

\begin{tabular}{|c|c|c|c|c|c|c|c|c|}
\hline Preparation Method & Batch & R6G $(\mu \mathrm{g})$ & Size & $\begin{array}{l}\text { Zeta-Potential } \\
(\mathrm{mV})\end{array}$ & $\begin{array}{c}\text { Yield } \\
(\%)\end{array}$ & $\begin{array}{l}\text { PVA Con- } \\
\text { tent }(\%)\end{array}$ & $\begin{array}{c}\text { Drug Loading } \\
\text { (\%) }\end{array}$ & $\begin{array}{c}\text { Entrapment } \\
\text { Efficiency (\%) }\end{array}$ \\
\hline \multirow{4}{*}{ Solvent Evaporation } & A & 0 & $274.0 \mathrm{~nm}$ & $-16.2 \pm 0.3$ & 86.51 & 1.33 & 0.93 & 56.47 \\
\hline & B & 0 & $303.9 \mathrm{~nm}$ & $-20.3 \pm 0.5$ & 85.40 & 2.14 & 1.15 & 69.93 \\
\hline & $\mathrm{C}$ & 100 & $472.5 \mathrm{~nm}$ & $-32.4 \pm 1.1$ & 86.92 & 1.13 & 0.80 & 48.79 \\
\hline & $\mathrm{D}$ & 100 & $351.8 \mathrm{~nm}$ & $-21.8 \pm 0.2$ & 88.94 & 0.80 & 0.94 & 57.16 \\
\hline \multirow{4}{*}{ Solvent Diffusion } & E & 0 & $7.27 \mu \mathrm{m}$ & $-22.5 \pm 0.4$ & 87.12 & 1.28 & 0.93 & 56.44 \\
\hline & F & 0 & $7.88 \mu \mathrm{m}$ & $-17.4 \pm 1.7$ & 75.17 & 1.25 & 1.30 & 79.51 \\
\hline & G & 100 & $7.55 \mu \mathrm{m}$ & $-26.6 \pm 2.0$ & 81.97 & 0.54 & 1.07 & 64.97 \\
\hline & $\mathrm{H}$ & 100 & $8.47 \mu \mathrm{m}$ & $-23.7 \pm 2.7$ & 71.78 & 0.47 & 1.05 & 64.14 \\
\hline
\end{tabular}

may be due to precipitation of the insulin-protamine complex out of the solution in a non-homogenous and fast fashion, which contributed to the formation of large particles. Based on particle size and size distribution, the solvent evaporation method was considered the better method for preparation of nanoparticles for insulin-protamine complexes. All batches of micro- and nanoparticles carried a negative charge, with similar zeta-potential (all below $-16.2 \mathrm{mV}$ ).

Inclusion of protamine in the formulation achieved insulin loading of about $1 \%$ and entrapment efficiency above $50 \%$, in comparison to the insulin entrapment efficiency of less than $2 \%$ when nanoparticles were formulated without protamine. This suggests that the formation of insulinprotamine complex can reduce insulin solubility in the outer water phase and thus substantially improve insulin loading and entrapment efficiency in the particles. This finding is in agreement with the published study of Cui et al. in which insulin-phospholipid complex was employed to enhance the entrapment of insulin in PLGA nanoparticles [13].

Insulin is reported to be stable under static conditions but unstable under stirring, transforming completely into insoluble products after only $24 \mathrm{~h}$ [15]. Therefore the in vitro release study of insulin-loaded micro- and nanoparticles was performed under static conditions. The release profile of the PLGA nanoparticles prepared with the solvent evaporation method is presented in (Fig. 2). Nanoparticles containing complexed insulin showed a burst release of $15-25 \%$ of loaded insulin at $1 \mathrm{~h}$, followed by a slow release. About 38$50 \%$ of loaded insulin was released over 7 days. Although Batch D, which had slightly smaller particles and therefore a larger surface area, showed a higher burst release (about $25 \%$ of the total loaded drug) than Batch $\mathrm{C}$ (about $15 \%$ of the total loaded drug), both batches exhibited similar release profiles over 7 days. Hence the different initial burst release could also be the result of the presence of different amounts of 'mother liquor' in the system. The subsequent first order release profile was probably due to insulin diffusion from the matrix at this stage of release, which correlated well with reported release kinetics for matrix type nanoparticles [20, 21].

The initial burst release can be attributed to the insulin located at the surface of the particles. In the FESEM images, it was observed that some nano-crystal structures adsorbed

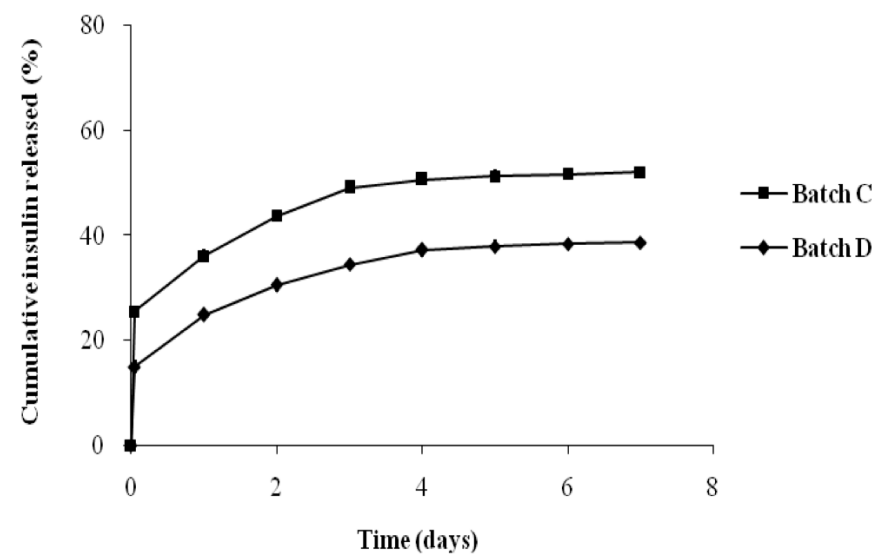

Fig. (2). Cumulative release of insulin from PLGA nanoparticles prepared by insulin-protamine complex and solvent evaporation method. Each data point represents the mean \pm SD $(n=3)$.

on the surface of the PLGA nanoparticles (Fig. 1a), which could be the complex of insulin-protamine. The remainder of the unreleased insulin was assumed to be entrapped within the PLGA nanoparticles as a stable complex with protamine. This hypothesis was confirmed by the analysis of unreleased insulin in the nanoparticles. Nanoparticles that completed the release study were dissolved in acetonitrile and unreleased insulin extracted by $0.01 \mathrm{M} \mathrm{HCl}$ and analysed by HPLC. The total amount of insulin detected (released and unreleased) was $89-92 \%$ of loaded insulin. The unaccounted for $8-11 \%$ could result from insulin degradation and assay variation in experimental operations. Further release of the remainder of insulin (about $40-50 \%$ of loaded insulin) would depend on the degradation of PLGA.

Due to the sustained insulin release exhibited by the insulin-protamine complex formulation, we hypothesized that a similar phenomenon would be observed when the solvent diffusion method was applied to prepare nanoparticles under mild conditions. However results obtained for PLGA particles loaded with insulin-protamine complex prepared by the solvent diffusion method were not as expected. The size of particles obtained was greater than one micron. Surprisingly the in vitro release showed a higher burst release of $76-78 \%$ during the first hour followed by a very slow release of 6$12 \%$ over the next 7 days (Fig. 3). This suggests that most of the insulin was located on the surface rather than incorporated within the particles. 


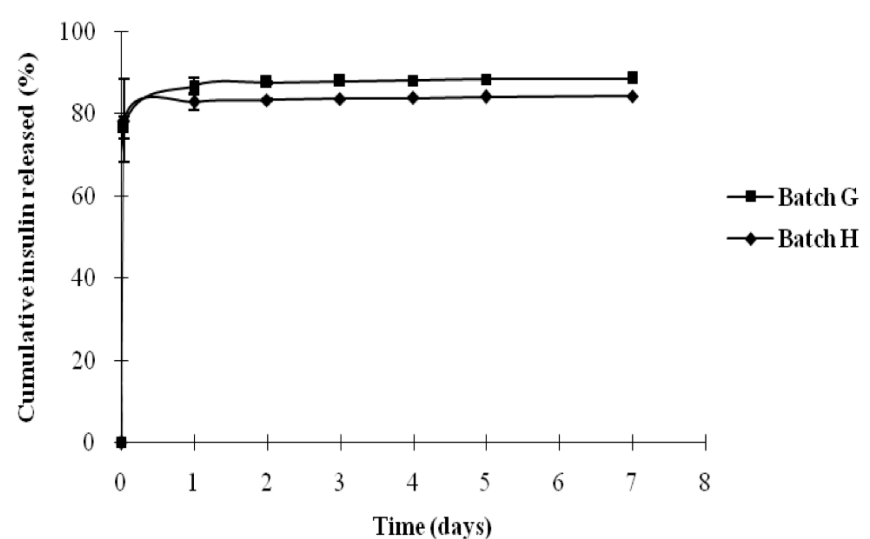

Fig. (3). Cumulative release of insulin from PLGA microparticles prepared by insulin-protamine complex and solvent diffusion method. Each data point represents the mean $\pm S D(n=3)$.

From the FESEM image (Fig. 1b), many nano structures can be seen on the surface of the particles, which could be the insulin-protamine complex, but further study is required to confirm this hypothesis. During the manufacturing process the internal water phase containing the insulin-protamine complex was miscible with the organic phase which consisted of ethanol/acetone $(4 / 6, \mathrm{v} / \mathrm{v})$. The volume ratio of internal water phase to organic phase (w/o) was 1:6.7 and such a large water fraction dramatically reduced the solubility of PLGA in the mixture of internal water phase and organic phase. Hence the polymer precipitated immediately upon addition of the internal water phase into the organic solvent of ethanol/acetone containing PLGA, which led to the formation of microparticles. As a result of solvent miscibility, the insulin-protamine complex may have migrated to the interface between the organic and water phases, resulting in accumulation of insulin on the surface, rather than incorporation inside the PLGA particles. This would explain the large burst release observed followed by a slow release of insulin observed. Similarly for the formulation prepared by the solvent evaporation method exhibited very slow release of insulin that can be attributed to the stable complex formed between insulin and protamine within the polymer matrix. About $6.5-9.1 \%$ of loaded insulin remained within the PLGA particles after 7 days release (as determined by degradation of the polymer matrix).

In order to study the particle distribution on/in the skin, R6G was incorporated into the particles as a marker for fluorescence microscopy to permit visualization of micro- and nanoparticle permeation in the skin. The presence of R6G slightly increased the mean diameter of insulin loaded microand nanoparticles, while the insulin loading and entrapment efficiency were unchanged. These results indicate that although R6G could compete with insulin to be incorporated inside particles, it did not appreciably alter the physicochemical characteristics at the concentration used (Table 3 ) and is therefore a suitable fluorescent label.

Analysis of the PVA content in the freeze dried product showed a low PVA content $(<3 \% \mathrm{w} / \mathrm{w})$ in all batches of insulin loaded micro- and nanoparticles (Table 3). This indicates that the involvement of PVA in the formulation had a negligible effect on the yield of insulin loaded particles and that the purification method employed in this study efficiently to remove the bulk of free PVA.

\section{Skin Deposition Study: Distribution of Insulin Loaded Nanoparticles}

The fluorescent images of different batches of particles in the skin deposition study are presented in (Fig. 4). As controls, we applied R6G solution and water to the skin. R6G solution generated a homogenous red background corresponding to the free probe. This strong fluorescence in the stratum corneum was expected as $\mathrm{R} 6 \mathrm{G}$ is lipophilic and can readily diffuse into the lipophilic regions of the skin. A weak fluorescence was observed from the skin with the water application, representing the intrinsic fluorescence of the skin (Fig. 4a).

The largest microparticles (batch $\mathrm{G}, 7.55 \mu \mathrm{m}$ ) prepared by insulin-protamine complex plus solvent diffusion methods were seen only on the skin surface with no deposition within the skin over the $24 \mathrm{~h}$. However, nanoparticles prepared from insulin-protamine complex and solvent evaporation (batch $\mathrm{C}$ and $\mathrm{D}, 472.5 \mathrm{~nm}$ and $351.8 \mathrm{~nm}$ ) were deposited within the skin at the appendages such as hair follicles, sweat glands and sebaceous glands (Fig. 4b) showing batch $\mathrm{C}$ nanoparticles deposited around hair follicles. These results indicate that particle size has a significant influence on skin permeation of a particulate carrier as has been previously reported [4, 22]. It was observed that the majority of nanoparticles remained on the surface of the skin (Fig. 4c) and desquamation was occurring with the stratum corneum, a phenomena that was also shown in the study of Cross et al. [6]. Those particles that appeared in the region between the stratum corneum and viable epidermis were more likely to be caused by the cutting procedure.

It is evident that no detectable amount of insulin or R6G penetrated through the skin to the receptor in a period of 24 $\mathrm{hr}$. This indicates that insulin and PLGA micro/nanoparticles skin penetration was minimal if any. These observations are in agreement with previously reported data obtained utilizing various types of polymer $[1,4]$ and inorganic particle formulations $[5,6,23]$.

For example, following repeated application of an $\mathrm{o} / \mathrm{w}$ emulsion containing micronised $\mathrm{TiO}_{2}$ particles (crystal size approx $17 \mathrm{~nm}$ ) to the forearms of volunteers over several days, only the upper stratum corneum and hair follicles showed any evidence of particle penetration [23]. A recent study of pig skin exposed to microfine $\mathrm{ZnO}$ (mean primary particle size $80 \mathrm{~nm}$ ) and $\mathrm{TiO}_{2}$ (siliconised needle-like particles $30-60 \times 10 \mathrm{~nm}$ ) sunscreen formulations suggested that these particle types did not penetrate beyond the top few layers of the stratum cornuem [5]. More recently, Cross and co-workers [6] reported a lack of $\mathrm{ZnO}$ nanoparticles $(30 \mathrm{~nm})$ in the lower stratum corneum and epidermis when $\mathrm{ZnO}$ particles were applied to the human skin set in Franz-type diffusion cells over a $24 \mathrm{hr}$ exposure period. These observations were supported by the work of Alvarez-Róman et al. [4] who visualized the skin penetration and distribution of 20 and 200 $\mathrm{nm}$ fluorescent polystyrene nanoparticles applied to porcine ear skin by CLSM. Nanoparticles accumulated within the hair follicles, with no direct penetration via the stratum corneum detected. Lademann et al. [1] reported a similar finding with their $320 \mathrm{~nm}$ fluorescent particles, but also demonstrated substantially enhanced distribution into the hair follicles when the formulation was applied with massage of the 
(a)

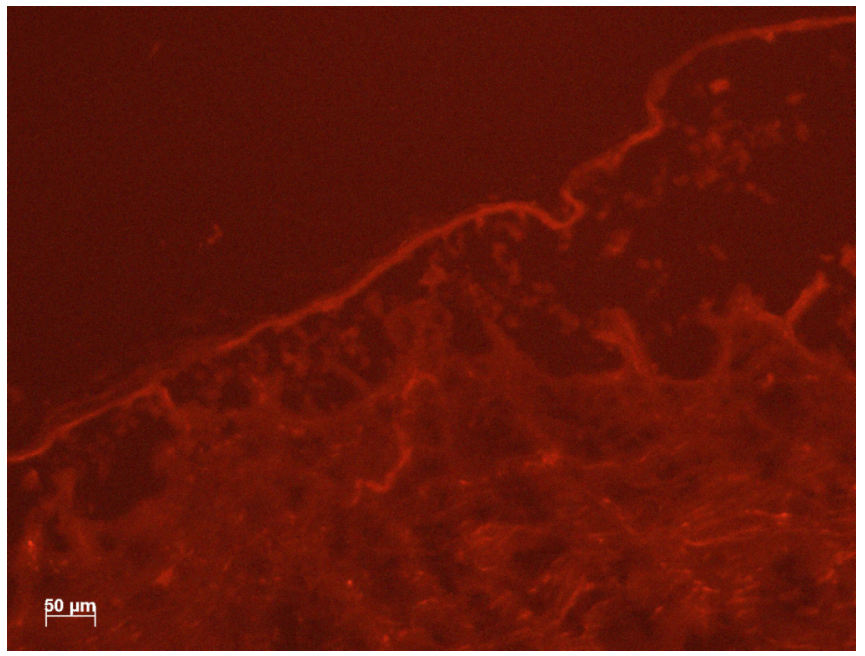

(b)

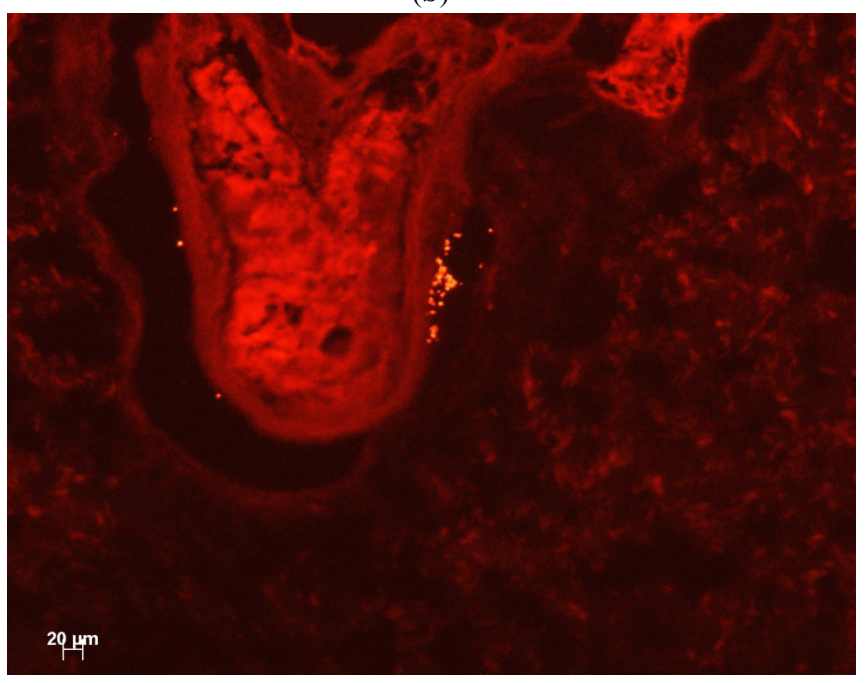

(c)

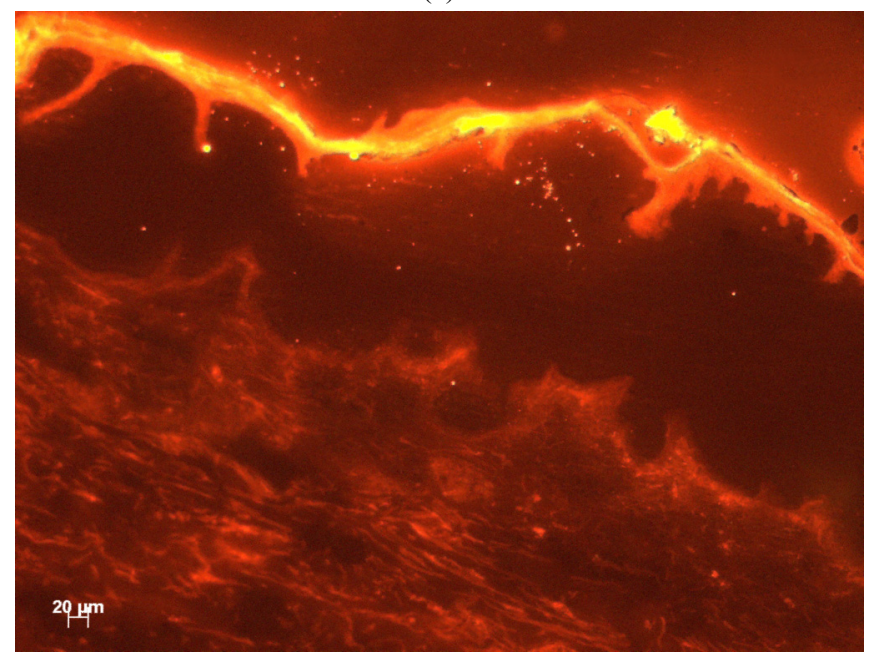

Fig. (4). (a) Fluorescence micrographs of vertically sliced human skin in water. (b) Fluorescence micrographs of vertically sliced human skin after $24 \mathrm{hr}$ permeation study of particles $(472.5 \mathrm{~nm})$ prepared by solvent evaporation method (Batch C). (c) Fluorescence micrographs of vertically sliced human skin after $24 \mathrm{hr}$ permeation study of particles $(351.8 \mathrm{~nm})$ prepared by solvent evaporation method (Batch D). skin. As topical products are normally rubbed on the skin it is likely that targeted delivery of particles to the hair follicles would be greater than that reported in this and the previously reported studies as a static application protocol is generally used for in vitro skin diffusion studies. These studies demonstrate the ability of nanoparticles to target delivery of their payload to the skin appendages with the possibility of subsequent controlled release and availability within the skin. This could be a useful delivery method for drugs and peptides that have poor stability and permeation within the skin.

\section{CONCLUSIONS}

By forming a complex between insulin and protamine, a significant amount of insulin was incorporated into PLGA particles and these particles provided sustained release of insulin over 7 days with good insulin stability. Entrapment efficiency of around $50 \%$ was achieved. In the skin deposition study, fluorescence microscopy images indicated that insulin loaded microparticles with size above $7 \mu \mathrm{m}$ remained on the surface of the skin, whilst nanoparticles $(<1 \mu \mathrm{m})$ were seen within the skin and were associated primarily with the appendages. The results of this study indicate that PLGA particulate carriers $(<1 \mu \mathrm{m})$ are a promising peptide delivery system for targeting the skin appendages including hair follicles, sebaceous and sweat glands.

\section{ACKNOWLEDGMENTS}

This project was partly funded by an ARC-linkage grant LP0453964 and the Western Australian Biomedical Research Institute (WABRI) of Curtin University of Technology. The authors wish to thank Dr Xia Lou, formerly of the Lions Eye Institute (WA) for her assistance with the cryomicrotome and University of Western Australia for FESEM analysis.

\section{REFERENCES}

[1] Lademann, J.; Richter, H.; Teichmann, A.; Otberg, N.; BlumePeytavi, U.; Luengo, J.; Weiss, B.; Schaefer, U. F.; Lehr, C. M.; Wepf, R.; Sterry, W., Eur. J. Pharm. Biopharm., 2007, 66, 159.

[2] Namjoshi, S.; Cacetta, R.; Benson, H. A. E., J. Pharm. Sci., 2007, DOI: 10. 2002/jps.21198.

[3] Kohli, A. K.; Alpar, H. O., Int. J. Pharm., 2004, 275, 13.

[4] Alvarez-Roman, R.; Naik, A.; Kalia, Y. N.; Guy, R. H.; Fessi, H., J. Control. Release, 2004, 99, 53.

[5] Gamer, A. O.; Leibold, E.; van Ravenzwaay, B., Toxicol. in Vitro, 2006, 20, 301.

[6] Cross, S. E.; Innes, B.; Roberts, M. S.; Tsuzuki, T.; Robertson, T. A.; McCormick, P., Skin Pharmacol. Physiol., 2007, 20, 148.

[7] des Rieux, A.; Fievez, V.; Garinot, M.; Schneider, Y.-J.; Preat, V., J. Control. Release, 2006, 116, 1 .

[8] Vert, M., Crit. Rev. Ther. Drug Carrier Syst., 1986, 2, 291.

[9] Bilati, U.; Allemann, E.; Doelker, E., Eur. J. Pharm. Biopharm., 2005, 59, 375 .

[10] Zambaux, M. F.; Bonneaux, F.; Gref, R.; Maincent, P.; Dellacherie, E.; Alonso, M. J.; Labrude, P.; Vigneron, C., J. Control. Release, 1998, 50,31 .

[11] Govender, T.; Stolnik, S.; Garnett, M. C.; Illum, L.; Davis, S. S., J. Control. Release, 1999, 57, 171.

[12] Yoo, H. S.; Park, T. G., J. Pharm. Sci., 2004, 93, 488.

[13] Cui, F.; Shi, K.; Zhang, L.; Tao, A.; Kawashima, Y., J. Control. Release, 2006, 114, 242 .

[14] Dalwadi, G.; Benson, H. A. E.; Chen, Y., Pharm. Res., 2005, 55, 2152.

[15] Rosa, G. D.; Iommelli, R.; La Rotonda, M. I.; Miro, A.; Quaglia, F., J. Control. Release, 2000, 69, 283.

[16] Moslemi, P.; Najafabadi, A. R.; Tajerzadeh, H., J. Pharm. Biomed. Anal., 2003, 33, 45. 
[17] Uchida, T.; Yagi, A.; Oda, Y.; Nakada, Y.; Goto, S., Chem. Pharm. Bull. (Tokyo), 1996, 44, 235.

[18] Uchida, T.; Nagareya, N.; Sakakibara, S.; Konishi, Y.; Nakai, A.; Nishikata, M.; Matsuyama, K.; Yoshida, K., Chem. Pharm. Bull. (Tokyo), 1997, 45, 1539.

[19] Defelippis, M. R.; Akers, M. J., Peptides and proteins as parenteral suspensions: an overview of design, development, and manufacturing considerations. In Pharmaceutical formulation development of peptides and proteins, Frokjaer, S.; Horgaard, L., Eds. Taylor\&Francis: London, 2000; pp 113-44.
[20] Radwan, M. A., Drug. Dev. Ind. Pharm., 1995, 21, 2371.

[21] Fresta, M.; Puglisi, G.; Giammona, G.; Cavallaro, G.; Micali, N.; Furneri, P. M., J Pharm. Sci., 1995, 84, 895.

[22] du Plessis, J.; Ramachandran, C.; Weiner, N.; Muller, D. G., Int. J. Pharm., 1994, 103: 277.

[23] Lademann, J.; Weigmann, H. J.; Rickmeyer, C.; Barthelmes, H.; Schaefer, H.; Mueller, G.; Sterry, W., Skin Pharmacol. Appl. Skin Physiol., 1999, 12, 247. 